\title{
Colonization and Infection
}

\author{
L. Silvestri, G. Mino, H.K.F van SAene
}

\section{Introduction}

Physiologically, internal organs such as lower airways and bladder are sterile. However, colonization of lower airways and bladder by potentially pathogenic micro-organisms (PPMs) is common in critically ill patients on the intensive care unit (ICU) [1]. Colonization of the internal organs generally follows the impaired carriage defense of the digestive tract, which promotes carriage and overgrowth of PPMs (see Chapter 2), and the impaired defenses of the host against colonization, due to illness severity. Failure to clear colonizing microorganisms from the internal organs invariably leads to high concentrations of PPMs, predisposing to invasion. The host mobilizes both humoral and cellular defense systems to hinder the invading micro-organisms. However, infection will require not only invasion, but also severity of the underlying disease, which jeopardizes immunocompetence.

The aims of this chapter are to define the concepts of colonization/infection and to describe the defense mechanisms of the host and the interventions for control of colonization/infection.

\section{Definitions}

\section{Colonization}

Colonization is defined as the presence of a micro-organism in an internal organ that is normally sterile (e.g., lower airways, bladder), without an inflammatory response of the host (Fig. 1). Diagnostic samples such as lower airway secretions, wound fluid, and urine generally yield $<10^{5}$ colony forming units (CFU) of potential pathogens per milliliter of diagnostic sample. In general, 


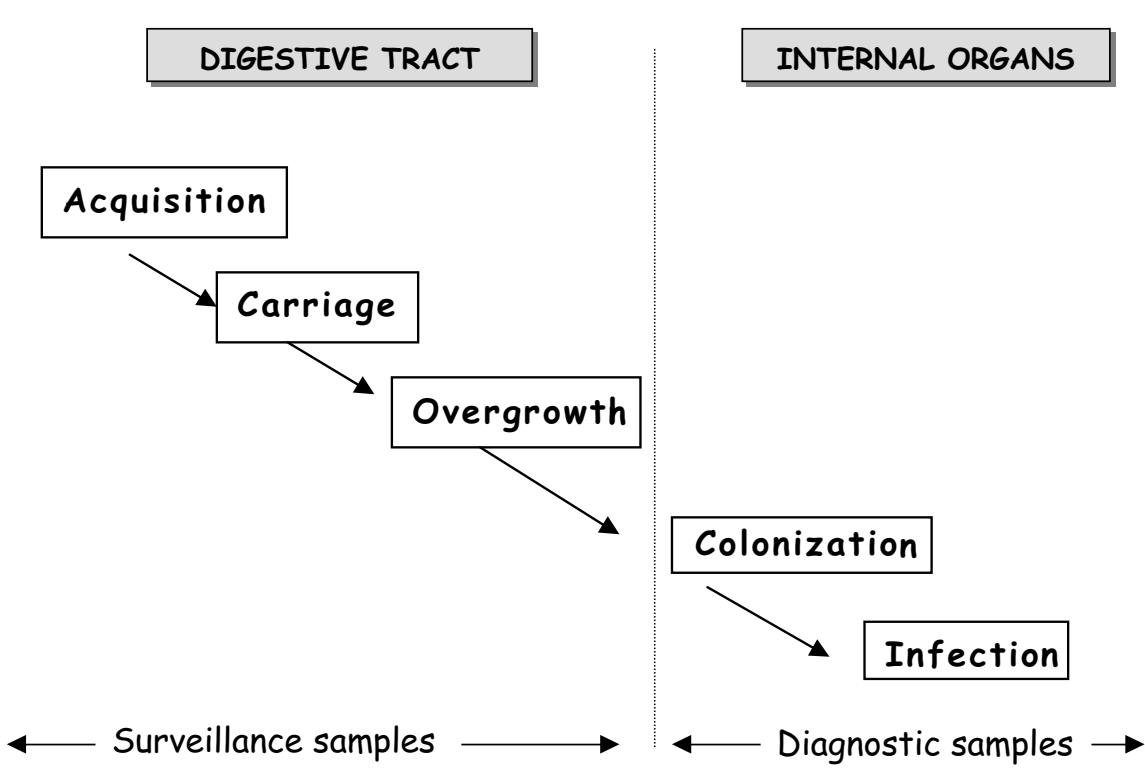

Fig. 1. The slippery slope of the pathogenesis of infection in critically ill patients (from Silvestri L, Lenhart FP, Fox MA (2001) Prevention of intensive care unit infections. Curr Anaesth Crit Care 12:34-40, with permission)

Acquisition develops if only one surveillance sample is positive for a potentially pathogenic micro-organism (PPM) that differs from the previous and following isolates. Acquisition refers to the transient presence of a micro-organism (usually in the oropharynx and gut), whilst carriage is a persistent phenomenon

Carriage or carrier state is the patient's state where the same bacterial strain is isolated from at least two surveillance samples (saliva, gastric fluid, feces, throat and rectal swabs) in any concentration over a period of at least 1 week

Overgrowth is defined as $\geq 10^{5} \mathrm{CFU} / \mathrm{ml}$ of saliva, gastric fluid, or g of feces, and is nearly always present in the critically ill ICU patient with impaired gut motility

Colonization is the presence of a PPM in an internal organ that is normally sterile (e.g,. lower airways, bladder) without an inflammatory host response. The diagnostic sample yields $<10^{5} \mathrm{CFU} / \mathrm{ml}$ of diagnostic sample

Infection is a microbiologically proven clinical diagnosis of inflammation. Apart from the clinical signs of infection the diagnostic sample obtained from the internal organ contains $\geq 10^{5} \mathrm{CFU} / \mathrm{ml}$ or is positive in the case of blood, cerebrospinal and pleural fluid Surveillance samples are samples from body sites where PPMs are carried, such as digestive tract, and skin lesions (tracheotomy, wounds, pressure sores). A surveillance set comprises throat and rectal swabs taken on admission and afterwards twice weekly, e.g., on Monday and Thursday. The purpose of surveillance samples is the determination of the microbiological endpoint of the level of carriage of PPMs

Diagnostic samples are samples from internal organs that are normally sterile such as lower airways, blood, and bladder. The aim of diagnostic samples is clinical, i.e., to microbiologically prove a diagnosis of inflammation, both generalized or local 
only a few leukocytes are present in colonized internal organs on a semiquantitative scale of $+=$ few, $2+=$ moderate, and $3+=$ many leukocytes $[2,3]$.

The term colonization should be distinguished from carriage. Carriage is defined when the same strain is isolated from at least two surveillance samples (saliva, gastric fluid, feces, throat and rectal swabs) in any concentration over a period of at least 1 week (Fig. 1). Carriage and colonization are two different stages in the pathogenesis of endogenous infection in ICU patients. The first stage is almost always the oropharyngeal and gastrointestinal carrier state followed by overgrowth. Once the PPMs are present in high concentrations, in general $\geq 10^{5}$ of potential pathogens per $\mathrm{ml}$ of saliva and/or g of feces, they migrate into the sterile internal organs in order to colonize the lower airways and bladder. Unfortunately the term colonization is often used to cover both stages of carriage and colonization. Grouping together carriage and colonization may be misleading in interpreting the efficacy of antimicrobial interventions and risk factors for infection in ICU patients. For example, oral fluconazole was compared with oral nystatin in liver transplant patients to evaluate the impact on bladder colonization and rectal carriage [4]. Fluconazole is absorbable and hence sterilizes the bladder, whilst nystatin, which is not absorbed, does not have any impact on bladder colonization. However, fluconazole was no better in reducing carriage than nystatin. The authors reported that Candida colonization was significantly reduced in the fluconazole group following the inclusion of the data of the urine cultures. The Geneva group developed a colonization index to predict candidemia [5]. Both surveillance (throat and stomach) and diagnostic (lower airways, bladder, and wound) samples were considered to identify patients at high risk of severe Candida infections. When half the samples yielded Candida in heavy growth density, the chance of severe Candida infection was significantly higher than in patients who only had $20 \%$ of samples positive for Candida in high concentrations. We believe that this index is not very useful from a clinical point of view, as the authors were unable to recommend the timing of antifungal therapy. The normal physiological condition of tracheal aspirate, urine, and wound fluid is sterility. These diagnostic samples can only be kept free of yeasts if those microorganisms are absent or present in low concentration in throat, stomach, and gut. From a prevention point of view, enteral antifungals should be started as soon as possible, particularly if throat and rectal swabs show yeast in overgrowth.

\section{Infection}

Infection is a microbiologically proven clinical diagnosis of inflammation, local and/or generalized. This includes not only clinical signs, but also the presence of at least a moderate (2+) number of leukocytes and micro-organisms of $\geq 10^{5}$ $\mathrm{CFU} / \mathrm{ml}$ in diagnostic samples obtained from an internal organ, or the isolation 
of a micro-organism from blood, cerebrospinal fluid, or pleural fluid. Sepsis is defined as clinical signs of generalized inflammation caused by micro-organisms and/or their products. Septicemia is sepsis combined with a positive blood culture (Fig. 1) [2]. In some studies of infection rates in liver transplant patients, microbiological proof was not even required, and vague terms, including "a positive culture from a $\mathrm{T}$ tube" [6] or "infected bile" [7], were used to define infections of wounds and bile.

\section{Samples}

Diagnostic samples are samples from internal organs that are normally sterile, such as the lower airways, bladder, and blood. They are obtained when clinically indicated and allow the diagnosis of colonization and infection [2].

Surveillance samples are samples from body sites where the potential pathogens are carried, i.e., the digestive tract, and skin lesions (tracheotomy, wounds, pressure sores). Generally, a set of surveillance samples consists of throat and rectal swabs taken on admission of the patient to the ICU and twice weekly, thereafter. The purpose of surveillance samples is the determination of the microbiological endpoint of the level of carriage of PPMs. They are not useful for diagnosing infection of internal organs, as diagnostic samples are required for this purpose [2].

\section{Internal Organs in Health and Disease}

\section{Carriage}

Micro-organisms are carried in the oropharynx, gut, and vagina. Micro-organisms present in healthy people belong to the "normal flora". They are mainly anaerobes and aerobes of the indigenous flora, together with "community" micro-organisms, such as Streptococcus pneumoniae, Staphylococcus aureus, Haemophilus influenzae, Moraxella catarrhalis, Escherichia coli, and Candida albicans. The "opportunistic" or hospital micro-organisms are uncommon in healthy people, and may be only transiently present [8], whilst disease promotes oropharyngeal and gastrointestinal carriage of these abnormal micro-organisms. They include eight aerobic Gram-negative bacilli (AGNB) (e.g., Klebsiella, Proteus, Morganella, Enterobacter, Citrobacter, Serratia, Acinetobacter, and Pseudomonas spp.), and methicillin-resistant $S$. aureus (MRSA). Approximately one-third of patients with an underlying chronic condition such as diabetes, alcoholism, or chronic obstructive pulmonary disease (COPD) are likely to demonstrate abnormal bacteria in their oropharynx and gut [8]. Moreover, previously healthy patients admitted to the ICU and requiring long-term ventila- 
tory support due to an acute insult, such as (surgical) trauma, pancreatitis, or acute liver failure, may become carriers of abnormal hospital flora in their digestive tract (Chapter 2).

\section{Colonization and Infection}

Secretions from internal organs such as the lower airways, sinuses, middle ear, lachrymal gland, and urinary tract of healthy individuals are normally sterile. Colonization of internal organs can occur with the two types of PPMs, community, including S. pneumoniae and $H$. influenzae, and "abnormal" opportunistic PPMs such as Klebsiella and Pseudomonas species. Three examples illustrate the concept of colonization followed by infection.

1. Elderly people cared for in a nursing home carry S. pneumoniae and $H$. influenzae in their oropharynx. During winter months elderly people are at high risk of developing the flu. The flu virus destroys the cilia and causes systemic immunosuppression. Colonization of the lower airways with $S$. pneumoniae and $H$. influenzae invariably occurs in this population during the flu epidemic. If these patients do not receive a short course of commonly used antibiotics, colonization of the lower airways often progresses to pneumonia associated with high mortality. A similar pattern has been described for trauma patients $[9,10]$.

2. COPD patients with a forced expiratory volume in $1 \mathrm{~s}\left(\mathrm{FEV}_{1}\right)<50 \%$ are oropharyngeal carriers of both types of flora, including $H$. influenzae and AGNB [11]. The severity of their underlying lung disease promotes colonization of the lower airways with oral flora, including "community" and "hospital" bacteria. The presence of bacteria in the lower airways or colonization is proinflammatory and may result in a range of important effects on the lung. These include activation of host defenses with release of inflammatory cytokines, such as interleukin-8 [12] and subsequent neutrophil recruitment, mucus hypersecretion, impaired mucociliary clearance, and respiratory cell damage [13]. Bacterial colonization of lower airways in COPD patients modulates the character and frequency of exacerbations [14], and is associated with greater airway inflammation and an accelerated decline in $\mathrm{FEV}_{1}$ [15]. An acute exacerbation of their underlying condition may require intubation and ventilation on the ICU. The immediate administration of an adequate antimicrobial that is active against $H$. influenzae and AGNB such as Klebsiella species is required in order to prevent infection of the lower airways (see Chapter 19).

3. A patient who is transferred from another hospital or ward into the ICU needing ventilatory support often carries abnormal flora, including MRSA or Pseudomonas species, due to the underlying disease. The acute deterioration of their underlying disease requires intubation leading to colonization 
of the lower airways with hospital flora. Colonization may develop into infection depending on the level of the immunosuppression of the patient. The experience that a delay in immediate adequate antimicrobial treatment increases mortality prompted the American Thoracic Society to advocate empirical treatment, including vancomycin and ciprofloxacin, to cover MRSA and Pseudomonas [16].

\section{Patient's Defenses Against Colonization and Infection}

\section{Defense Against Colonization of the Internal Organs [17]}

The first line of defense against carriage of PPMs in the digestive tract has been described in detail in Chapter 2. "Abnormal" carriage of AGNB and MRSA inevitably leads to overgrowth of these bacteria in a patient who requires mechanical ventilation following deterioration of the underlying disease. The multiple factors and interventions that promote overgrowth are discussed in Chapter 2. Intestinal overgrowth of abnormal flora promotes and maintains systemic immunoparalysis via liver macrophage activation [18], and is considered an independent risk factor for colonization and infection of internal organs [19-21]. PPMs may migrate from the digestive tract towards lower airways or bladder (endogenous colonization) or may be introduced directly into the internal organ from an "external" source, both animate or inanimate (exogenous colonization). Six clearing factors are present in these internal organs. In the lower airways these are the following [22] (Fig. 2).

Integrity of anatomy. The endotracheal tube damages the mucosa and promotes adherence of micro-organisms.

Intactness of physiology. Inhaled particles or micro-organisms have to survive and penetrate the aerodynamic filtration system of the tracheobronchial tree. The airflow is turbulent, causing micro-organisms to affect the mucosal surfaces. Humidification also causes hygroscopic organisms to increase in size, thereby aiding trapping. Mucosal surface adhesins are known to mediate adherence of bacteria to host extracellular matrix components, such as collagen, fibrinogen, and fibronectin [23-25]. Fibronectin covers the cell surface receptors and thereby blocks the attachment of many micro-organisms. The mucociliary blanket transports the invading micro-organism out of the lung, and coughing aids this expulsion. In addition, the bronchial secretions contain various antimicrobial substances, such as lysozyme, and defensins. Once the microorganism reaches the alveoli, the alveolar macrophages and tissue histiocytes play an important role in protecting the host.

Motility of cilia in conjunction with mucus mechanically removes microorganisms reaching its surface. Airway hygiene depends largely on mucociliary 


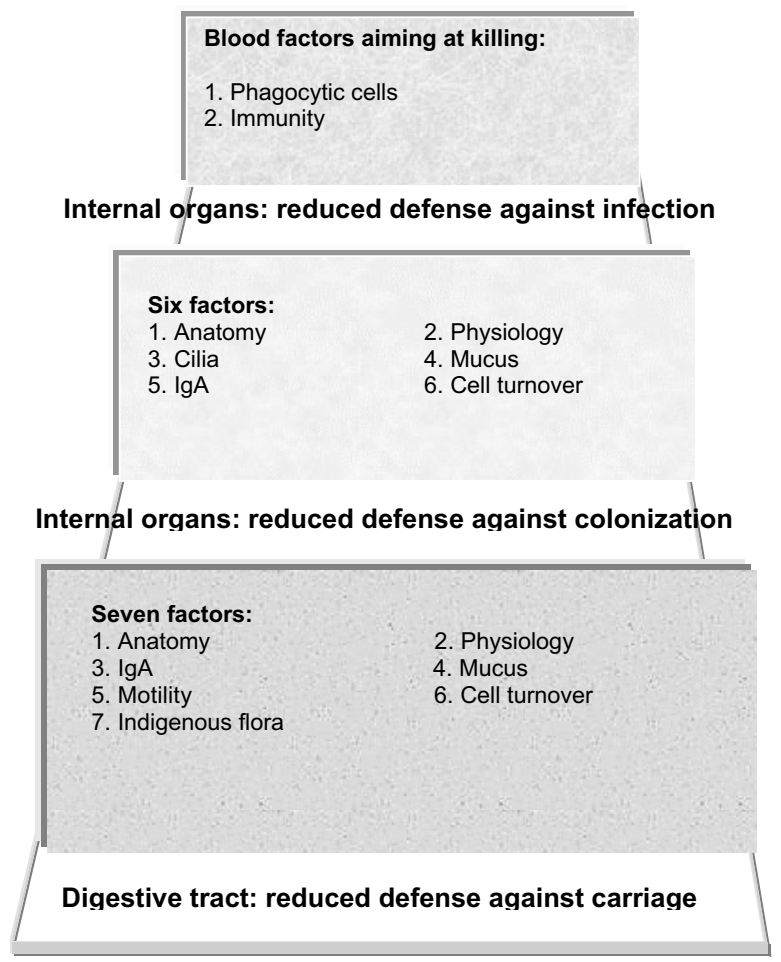

Fig. 2. Three hurdles of defense against carriage, colonization, and infection

clearance, which in turn depends upon the movement of viscoelastic mucus along the airway [26]. Cilia are small cell appendices aligned in the direction of the effective beat of the cilia, which move with a metachronal wave form. At the tip of each cilium there are protuberances that hook in the overlying mucus so as to propel it along the airway. Aspirated or breathed material sticks to this mucus, and it is thus cleared from the respiratory tract. A thin periciliary fluid made by products of alveolar and bronchial epithelium moves centrally, undergoing modifications in ion content and volume. Mucociliary clearance can be impaired by (1) genetic defects, e.g., primary ciliary dyskinesia, cystic fibrosis; (2) secondary ciliary dyskinesia due to artificial ventilation or toxins released by micro-organisms producing cytotoxic damage of epithelial cells (in this situation, micro-organisms may remain longer in the airways, causing colonization and infection); (3) abnormal physico-chemical properties of mucus, making it difficult to move it along the airway. A persistent host inflammatory response driven by cytokines fails to eliminate micro-organisms, which maintain the inflammatory process. 
Secretory immunoglobulin A in bronchial secretions coats micro-organisms to prevent adherence to mucosal cell receptors. Secretory IgA is the predominant immunoglobulin present in the respiratory tract, nasal secretions, saliva, tears, gastrointestinal fluids, and other mucous secretions. In addition IgA can neutralize toxin activity [27].

Mucosal cell turnover and desquamation eliminate adherent bacteria.

Similarly, six mechanisms are present to prevent fecal PPMs from colonizing the urinary tract [28] (Fig. 2).

Integrity of anatomy. The bladder mucosa acts as a barrier to eliminate invading micro-organisms.

Intact physiology. This aims to clear PPMs and prevent them from migrating form the rectal cavity into the urethra and finally into the bladder [29]. Extreme levels of osmolality, high urea concentration, and low $\mathrm{pH}$ levels are inhibitory for the growth of some bacteria causing urinary tract infection.

Urinary flow and micturition mechanically remove PPMs unless they are capable of adhering to epithelial cells of the urinary tract.

Mucus covers the bladder mucosa.

Secretory IgA present in the mucus prevents adherence of fecal bacteria.

Mucosal cell turnover promotes the elimination of PPMs already adhering to bladder mucosal cells.

\section{Defense Against Infection}

Failure to eliminate colonizing micro-organisms from the internal organs invariably leads to high concentration of $\geq 10^{5} \mathrm{PPMs}$, predisposing to invasion. The host mobilizes both humoral and cellular defense systems to hinder the invading micro-organisms. However, infection requires not only invasion but also critical illness, which jeopardize immunocompetence, and the treatment of which facilitates crossing the physical barriers (Fig. 2).

\section{Mechanisms of Colonization and Infection in ICU Patients}

There are two basic mechanisms of colonization and infection in ICU patients: migration and translocation, or transmural migration. Migration is the movement of live PPMs from one place, e.g., throat and gut where they are present in overgrowth, to other sites, in particular normally sterile internal organs. Migration is the main mechanism by which micro-organisms cause endogenous colonization/infection in ICU patients. The migration of micro-organisms contained in contaminated secretions from the oropharynx into the lower airways within few days of mechanical ventilation is considered to be the most-common 
route by which PPMs enter the lung and cause colonization and infection $[19,30$, 31]. The severity of the underlying disease causing impairment of clearance of PPMs is the main factor promoting colonization of the lower airways. The presence of the plastic endotracheal tube is invariably associated with mucosal lesions, which further enhance colonization. Finally, the progress towards infection depends on the immune status or defense capacity of the patient.

Potential pathogens may also cause colonization and subsequent infection, bypassing the stage of carriage and overgrowth, i.e., exogenous colonization/ infection. An example is a lower respiratory tract colonization/infection in a tracheotomized patient due to micro-organisms not previously carried in the throat and/or gut, but directly introduced into the internal organ following breaches of hygiene [32,33].

Translocation (or transmural migration) is defined as the ingress of oropharyngeal or gut PPMs through the mucosal lining of the alimentary canal into gut-associated lymphoid tissue (GALT), which includes macrophages in the mesenteric lymph nodes, liver, spleen, and blood. The GALT macrophages are generally effective in killing intestinal micro-organisms translocating from the gut in normally healthy people. In the case of impairment of gut function, such as in the critically ill patient, either with an anatomically intact gastrointestinal tract or altered intestinal mucosa, bacterial translocation can be followed by spreading into the systemic bloodstream, leading to sepsis and multiple organ failure [34]. Gut overgrowth of PPMs, in particular in the terminal ileum, is required for transmural migration or translocation [35]. The phenomenon of translocation has been described in surgical patients [21,36], patients with pancreatitis [20] and neutropenia [37], in surgical neonates and infants receiving parenteral nutrition [38], and in patients requiring intensive care, including mechanical ventilation [39].

\section{Control of Colonization/Infection}

Parenteral antimicrobials are required to sterilize internal organs such as lower airways, bladder, and blood. In patients who are successfully decontaminated, i.e., the throat and gut are free from potential pathogens including AGNB, $S$. aureus, and yeasts following selective decontamination of the digestive tract (SDD) [40], the diagnostic samples of lower airway secretions, blood, and urine are almost always sterile within 3 days of systemic antimicrobials [41]. The eradication of overgrowth in the throat and gut using SDD promotes sterilization of the internal organs, as the microbial load responsible for migration and translocation is reduced/lifted. Some micro-organisms, such as $S$. aureus sensitive and resistant to methicillin, $P$. aeruginosa, and yeasts, have an affinity for 
plastic devices, including the ventilation tube, the indwelling vascular devices, and the bladder catheter. Adhering micro-organisms may interfere with the sterilization process of the intravenously administered antimicrobials. If the lower airway secretions, blood, and urine are not sterile after 3 days of systemic administration of antimicrobials, removal and/or replacement of the foreign body is indicated. An additional step to control colonization/infection of the lower airways includes nebulization of the antimicrobials to increase the antimicrobial levels in the lower airways [42] (Chapter 23).

Table 1. Diagnostic samples: normal and abnormal values

\begin{tabular}{lcl}
\hline & Normal values & \multicolumn{1}{c}{ Abnormal values } \\
\hline Blood & Sterile & Any growth \\
Lower airway secretions & Sterile & $\begin{array}{l}\text { Any growth whether colonization or } \\
\text { infection, except viridans streptococci, } \\
\text { enterococci, coagulase-negative } \\
\text { staphylococci and yeasts }\end{array}$ \\
Urine & Sterile & $\begin{array}{l}\text { Any growth whether colonization } \\
\text { or infection, except enterococci, } \\
\text { coagulase-negative staphylococci }\end{array}$ \\
Wound & Sterile & $\begin{array}{l}\text { Any growth whether colonization or } \\
\text { infection, except coagulase-negative } \\
\text { staphylococci }\end{array}$ \\
Cerebrospinal fluid & Sterile & Any growth \\
\hline
\end{tabular}

\section{References}

1. Kerver AJH, Rommes JH, Mevissen-Verhage EAE et al (1987) Colonization and infection in surgical intensive care patients: a prospective study. Intensive Care Med 13:347-351

2. Sarginson RE, Taylor N, van Saene HKF (2001) Glossary of terms and definitions. Curr Anaesth Crit Care 12:2-5

3. A'Court CHD, Garrard CS, Crook D et al (1993) Microbiological lung surveillance in mechanically ventilated patients using non-directed bronchial lavage and quantitative culture. Q J Med 86:635-648

4. Lumbreras C, Cuervas-Mons V, Jara P et al (1996) Randomized trial of fluconazole versus nystatin for the prophylaxis of Candida infection following liver transplantation. J Infect Dis 174:583-588

5. Pittet D, Monod M, Suter PM, Frenk E, Auckentaler R (1994) Candida colonization and subsequent infections in critically ill surgical patients. Ann Surg 220:751-758 
6. Rayes N, Seehofer D, Hansen S et al (2002) Early enteral supply of Lactobacillus and fiber versus selective bowel decontamination: a controlled trial in liver transplant patients. Transplantation 74:123-128

7. Zwaveling JH, Maring JK, Klompmaker IJ et al (2002) Selective decontamination of the digestive tract to prevent postoperative infection: a randomized placebo-controlled trial in liver transplant patients. Crit Care Med 30:1204-1209

8. Mobbs KJ, van Saene HKF, Sunderland D, Davies PDO (1999) Oropharyngeal Gramnegative bacillary carriage. A survey of 120 healthy individuals. Chest 115:1570-1575

9. Sirvent JM, Torres A, Vidaur L et al (2000) Tracheal colonisation within $24 \mathrm{~h}$ of intubation in patients with head trauma: risk factor for developing early-onset ventilatorassociated pneumonia. Intensive Care Med 26:1369-1372

10. Ewig S, Torres A, El-Ebiary M et al (1999) Bacterial colonization patterns in mechanically ventilated patients with traumatic and medical injury. Incidence, risk factors and association with ventilator-associated pneumonia. Am J Respir Crit Care Med 159:188-198

11. Mobbs KJ, van Saene HKF, Sunderland D, Davies PDO (1999) Oropharyngeal Gramnegative bacillary carriage in chronic obstructive pulmonary disease: relation to severity of disease. Respir Med 93:540-545

12. Yamamoto C, Yoneda T, Yoshikawa M et al (1997) Airway inflammation in COPD patients assessed by sputum levels of interleukin-8. Chest 112:505-510

13. Sethi S, Murphy TF (2001) Bacterial infection in chronic obstructive pulmonary disease in 2000: state of the art. Clin Microbiol Rev 14:336-363

14. Patel IS, Seemungal TA, Wilks M et al (2002) Relationship between bacterial colonisation and the frequency, character, and severity of COPD exacerbations. Thorax 57:753-754

15. Wilkinson TMA, Patel IS, Donaldson GC, Wedzicha JA (2003) Airway bacterial load and FEV1 decline in patients with chronic obstructive pulmonary disease. Am J Respir Crit Care Med 167:1090-1095

16. Anonymous (1996) Hospital-acquired pneumonia in adults: diagnosis, assessment of severity, initial antimicrobial therapy, and preventing strategies. A consensus statement, American Thoracic Society. Am J Respir Crit Care Med 153:1711-1725

17. van Saene HKF, Damjanovic V, Alcock SR (2001) Basics in microbiology for the patient requiring intensive care. Curr Anaesth Crit Care 12:6-17

18. Marshall JC, Christou NV, Meakins JL (1988) Small-bowel bacterial overgrowth and systemic immuno-suppression in experimental peritonitis. Surgery 104:404-411

19. van Uffelen R, van Saene HKF, Fidler V et al (1984) Oropharyngeal flora as a source of colonizing the lower airways in patients on artificial ventilation. Intensive Care Med $10: 233-237$

20. Luiten EJT, Hop WCJ, Endtz HP et al (1988) Prognostic importance of Gram-negative intestinal colonization preceding pancreatic infection in severe acute pancreatitis. Intensive Care Med 24:438-445

21. de la Cal MA, Cerdà E, van Saene HKF et al (2004) Effectiveness and safety of enteral vancomycin to control endemicity of methicillin-resistant Staphylococcus aureus in a medical/surgical intensive care unit. J Hosp Infect 56:175-183

22. Manson CM, Summer WR, Nelson S (1992) Pathophysiology of pulmonary defence mechanisms. J Crit Care 7:42-56

23. Beachey EH (1981) Bacterial adherence. Adhesin-receptor interactions mediated the attachment of bacteria to mucosal surfaces. J Infect Dis 143:325-345

24. Peacock SJ, Foster TJ, Cameron BJ, Berend R (1999) Bacterial fibronectin-binding proteins and endothelial cell surface fibronectin mediate adherence of Staphylococcus aureus to resting human endothelial cells. Microbiology 145:3477-3486 
25. Mongodin E, Bajolet O, Cutrona J et al (2002) Fibronectin-binding proteins of Staphylococcus aureus are involved in adherence to human airway epithelium. Infect Immun 70:620-630

26. Cole P (2001) Pathophysiology and treatment of airway mucociliary clearance. Minerva Anestesiol 67:206-209

27. Hienzel FP (2000) Antibodies. In: Mandell GL, Bennett JE, Dolin R (eds) Mandell, Douglas and Bennett's Principles and practice of infectious diseases. Churchill Livingstone, Philadelphia, pp 45-67

28. Kass EH, Schneiderman LJ (1957) Entry of bacteria into the urinary tract of patients with implying catheters. N Engl J Med 256:556-557

29. Kunin CM, Evans C, Bartholomew D, Bates DG (2002) The antimicrobial defense mechanism of the female urethra: a reassessment. J Urol 168:413-419

30. Johanson WG Jr, Pierce AK, Sandford JP et al (1972) Nosocomial respiratory tract infections with Gram-negative bacilli: the significance of colonization of the respiratory tract. Ann Intern Med 77:701-706

31. Estes RJ, Meduri GU (1995) The pathogenesis of ventilator associated pneumonia. I. Mechanisms of bacterial transcolonization and airway inoculation. Intensive Care Med 21:365-383

32 de Latorre JF, Pont T, Ferrer A, Rossello J, Palomar M, Planas M (1995) Pattern of tracheal colonization during mechanical ventilation. Am J Respir Crit Care Med 152:1028-1033

33. Morar P, Makura Z, Jones A et al (2000) Topical antibiotics on tracheostoma prevent exogenous colonization and infection of lower airways in children. Chest 117:513-518

34. Sganga G, van Saene HKF, Brisinda G, Castagneto M (2001) Bacterial translocation. In: van Saene HKF, Sganga G, Silvestri L (eds) Infection in the critically ill: an ongoing challenge. Springer, Milan, pp 35-45

35. Husebye E (1995) Gastro-intestinal motility disorders and bacterial overgrowth. J Intern Med 237:419-427

36. Kane TD, Wesley Alexander J, Johannigman JA (1998) The detection of microbial DNA in the blood. A sensitive method for diagnosing bacteremia and/or bacterial translocation in surgical patients. Ann Surg 227:1-9

37. Tancrede CH, Andremont AO (1985) Bacterial translocation and Gram-negative bacteremia in patients with hematological malignancies. J Infect Dis 152:99-103

38. van Saene HKF, Taylor N, Donnell SC et al (2003) Gut overgrowth with abnormal flora: the missing link in parenteral nutrition-related sepsis in surgical neonates. Eur J Clin Nutr 57:548-553

39. Feltis BA, Wells CL (2000) Does microbial translocation play a role in critical illness? Curr Opin Crit Care 6:117-122

40. Baxby D, van Saene HKF, Stoutenbeek CP, Zandstra DF (1996) Selective decontamination of the digestive tract: 13 years on, what it is and what it is not. Intensive Care Med 22:699-706

41. Stoutenbeek CP, van Saene HKF, Miranda DR, Zandstra DF, Langrehr D (1986) Nosocomial Gram-negative pneumonia in critically ill patients. Intensive Care Med 12:419-423

42. Brown RB, Kruse JA, Counts GW (1990) Double-blind study of endotracheal tobramycin in the treatment of Gram-negative pneumonia. Antimicrob Agents Chemother $34: 269-272$ 